\title{
The Change and Development of Individual Project --Based on the Evolution of Code of Point Rhythmic Gymnastics 2017-2020
}

\author{
Yun Gu ${ }^{1, a}$, Bing Shi ${ }^{1, b}$, YuLiang Sun ${ }^{1, c}$ \\ ${ }^{1}$ School of Physical Education, Shaanxi Normal University, Xian 710119, Shaanxi China; \\ aaGuYungladys@hotmail.com, b378833269@qq.com,1010720@qq.com,
}

Keywords: rhythmic; gymnastics; rules; changes; development

\begin{abstract}
International Federation of Gymnastics constantly adjusted rules along with the development of competitive sports. This study proposed certain theoretical foundations for the future development of rhythmic gymnastics. Rhythmic gymnastics event is developing towards artistic, diversified and precise direction with higher requirement on athletes. Rhythmic gymnastics' scoring rule in the version of 2013-2016 and 2017-2020 of International Federation of Gymnastics were compared, literature review methods and summary and induction, to analyze the main characteristics of new rules, to predict the development of tendency of rhythmic gymnastics. A conclusion could be obtained: the rhythmic gymnastics development in China is at a rise phase, thus there is a large gap between rhythmic gymnastics of China and powerful nations worldwide in terms of art arrangement.
\end{abstract}

\section{Introduction}

Rhythmic gymnastics originated at the end of the 19th century in Europe and spread to Asia in the 1950s. It gradually became popular all over the world [1]. The Chinese Rhythmic Gymnastics Program started relatively late and is still under development. The rhythmic gymnastics requires athletes to use different light equipment (including ropes, hoops, balls, clubs, and ribbons) to perform various apparatus exercises (thrilling movements, rolling, throwing, etc.) and body movements (such as balance, rotation, jump, dance movements, etc.), music is accompanied by the characteristics of the athletes themselves, exercise based on elegant dance movements and diversified expressions project, as known as "ballet on the carpet." As the same of the Olympic, the change of rhythmic gymnastics rules were also for four years. At the end of each, according to the summary of the previous, the FIG would make reasonable changes to the scoring rules of the new. The change of scoring rules in each would determine the new direction for the development of rhythmic gymnastics in the future.

\section{Methods}

In this study, we collected and summarized the change of the rules and development trend of rhythmic gymnastics in China according to the literature. Through induction, the future development trend of Chinese rhythmic gymnastics is deduced. It compared and analyzed 2 edition rules on specific changes of content. Through video analysis, observation analyzed Chinese gymnastics athletes under the new rules change of completion.

\section{Results}

\section{1 analysis on the change of movements difficulty of individual sets $D$ group of new rhythmic gymnastics rules.}

"D" in the code of point of rhythmic gymnastics difficulty included four items: (BD) the difficulty of the body, dance step combination (S), (R) combined with dynamic rotation, and (AD) appliance difficulty. BD action refers to the jump, balance, and rotation , which were form "difficult table" by the code of points, which was usually denoted by symbols ( $\wedge, T, \delta)$. If a 
difficult action was completed, but no approval is obtained from the $\mathrm{D}$ group, then the action would not be evaluated and the judges would be deducted [2]. Since 2013-2016 version of the rules cut body difficulties form291 to 145 [3].

The degree of proficiency of rhythmic gymnast in the use of equipment reflected the level of the athletes, and the higher the proficiency, the more the value of the athletes can be reflected. Device difficulty proficiency was called "Appliance difficulties" in this period and gave a new definition. "AD" was not complete a simple without a thrilling instrument action, but must be completed by instrument synchronization with the body of a special technical difficulty, by at least a basic factors plus at least two standard form or of two basic factors plus one standard. It can be seen from table 1 the number of body difficulties gradually reduced.2013-2016 edition rules limit equipment skilled "AD" limit up to five in the complete set of movements, and in 2017-2020 edition rules "AD" at least one number, unlimited highest number. The number of Appliance difficulties has increased, encouraging the creation of breathtaking equipment.

Table 1 Difficulty Comparison between 2013 Edition Rules and 2017 Edition Rules

\begin{tabular}{lcc}
\hline \multicolumn{1}{c}{ Difficulty } & 2013 Edition Rules & 2017 Edition Rules \\
\hline Body Difficulties & At least 6,no more than 9 & At least 3,no more than 9 \\
Dance Step & At least 1 & At least 1 \\
Dynamic Rotation & No more than 3(R) & At least $1(\mathrm{R})$ \\
Appliance & No more than 5 & At least 1 \\
Difficulties & & \\
\hline
\end{tabular}

\subsection{Execution Changes in technical errors}

The completion of the referee requires contestants to complete the action in the process of each error must be punished, with the exception of the overall punishment. The deduction of technical errors during the adjudication process was also more and more harsh. Requires aesthetic and technical perfection, the biggest change in the technical error deduction in the 2017-2020 scoring rules was the elimination of a deduction of 0.3 (50\%) for each pair of left / right hand instruments used in a set of movements item. Table 2 illustrates that the new rule gives a new definition of the accuracy of the instrument parabola.

Table 2 Error Deducting Comparison between 2013 Edition Rules and 2017 Edition Rules

\begin{tabular}{ccc}
\hline Deduction & 2013 Edition Rules & 2017 Edition Rules \\
\hline-0.1 & Move 1 to 2 steps & Move 1 step \\
-0.3 & Move 3 or more steps & Move 2 steps \\
\hline
\end{tabular}

\subsection{Execution changes in artistic mistakes}

Rhythmic gymnastics would be gymnastics and art integration, not only reflected the essence of sport, but also showed the elegant art. Rhythmic gymnastics competitions including not only technical skills, expressiveness, but also the overall effect of the complete set of effects on athletes, such as soundtracks and competition clothing [4]. As can be seen from Table3, the 2017 version of the scoring rules added the criteria for body dynamics, connections, and rhythms to the score of artistic completion. Under the constant change and adjustment of the new rules, the rhythmic gymnastics was guided in the direction of "technology, artistry and perfection", leading the development of unique and elegant rhythmic gymnastics projects [5]. 
Table 3 Comparison of the Composition of Art between 2013 Edition Rules and 2017 Edition Rules

\begin{tabular}{lccccccc}
\hline & $\begin{array}{c}\text { Guiding } \\
\text { Idea }\end{array}$ & Rhythmic & $\begin{array}{c}\text { Body } \\
\text { Expression }\end{array}$ & Directions & $\begin{array}{c}\text { Dynamic } \\
\text { Changes }\end{array}$ & Connection & Musical \\
\hline 2013 & $\sqrt{ }$ & $\sqrt{ }$ & $\sqrt{ }$ & $\sqrt{ }$ & None & None & None \\
$\begin{array}{l}\text { Edition } \\
2017\end{array}$ & $\sqrt{ }$ & $\sqrt{ }$ & $\sqrt{ }$ & $\sqrt{ }$ & $\sqrt{ }$ & $\sqrt{ }$ & $\sqrt{ }$ \\
Edition & & & &
\end{tabular}

\subsection{Impact of scoring system changes on athletes}

The new scoring system and the new scoring rules had a direct impact on athletes after adjusting to the difficulty level, requiring athletes to use only those actions that they can perform perfectly with art and technical proficiency; Its own desired goals and high quality completed. Since the 2013-2016 version of the rules was canceled when the difficulty of completing the basic technology group and the device technology group combination, reducing the number of difficult actions, so you want to obtain high scores must increase the difficulty of a single action points for athletes, for athletes their own requirements are more stringent[6].

\section{Discussion}

Artistic performance of rhythmic gymnastics, technical use, and complicated dancing steps are the key factors to win this project. The levels of motor skills are based on the athlete's own athletic ability and the stability and gracefulness of the movement. The value orientation of the new scoring rules is that athletes only want to choose the action that she can complete completely. High quality artistry and proficiency are the future direction of rhythmic gymnastics technology. All the deviations from this expectation will be caused by Group E Referee. The new rules towards more diverse and artistic. Repeated device movements, or all device movements performed on the same plane without any change, would be considered as a lack of diversity and be penalized. Requires varied instrumentation techniques such as direction and trajectory to make use of the entire ground area in a varied and complete way to create different modes; such as the level and form of movement: athlete in the air, standing, on the ground, running, jumping, dancing Step, equipment movements such as: in different planes, different directions, with different body parts using different techniques to complete the equipment movements. Through this study, we hope that coaches and athletes should carefully understand the new rules while training in science, master the future development of rhythmic gymnastics and strive to make our rhythmic gymnastics enter the rhythmic gymnastics as soon as possible.

\section{Conclusion}

Rhythmic gymnastics as a performance-oriented project, artistic expression was one of the main scoring elements and plays an important role in the project. The development of rhythmic gymnastics project not only emphasizes "difficulty" but also pays more attention to "beauty", which is both "difficult" and "beautiful" to be the essence of rhythmic gymnastics. As a kind of social ideology, art is the spiritual demand in the field of humanity and the sporty aesthetic in the field of competitive sports. The full integration of the two forms the rhythmic gymnastics. The new rule states that every beat in a set of music must fit perfectly into all the movements, which means that a set of actions is not just a puzzle of a single difficulty, but a work that has a theme, thought, storyline. The new cycle rules so much emphasis on artistic expression, is a test of the ability of athletes to demonstrate artistic style; is a manifestation of coaching wisdom and aesthetic ability; is the embodiment of the audience eager to be influenced by the arts. 


\section{References}

[1] Min Wang, Yuan Chen. Development Trend and Socialization Function of "Diversified" Rhythmic Gymnastics. Journal of Wuhan Institute of Physical Education.2011,20 (1):81-83.

[2] Gymnastics Management Center of the State Sports General Administration.2013,2017 edition of the international gymnastics scoring rules.

[3] Hongmei Gao, Yun Yang. Changes of rules of rhythmic gymnastics and developmental strategies of rhythmic gymnastics in China. Journal of Wuhan Institute of Physical Education. 2011, 3 (10):99-100.

[4] Yang Zhang, Guisen Li. Under the new of rhythmic gymnastics complete set of innovative new ideas. Journal of Capital Institute of Physical Education.2015.1(27):70-74.

[5] Min Wang, Wen Yang, Zhennan Zhu. Development Trend of International Rhythmic Gymnastics from the Perspective of New period. Journal of Physical Education.2011,18 (3): 106-109.

[6] Judging from the Great Adjustment of Difficulties in the Version of 2009 to See the Development Trend of Rhythmic Gymnastics. Journal of Wisdom(12):162. 